\title{
Manifestações clínicas e desfecho gestacional ocasionados pela infecção por COVID-19
}

\author{
Clinical manifestations and gestational outcome caused by COVID-19 infection
}

Manifestaciones clínicas y evolución gestacional causadas por la infección por COVID-19

Gabriel Torga Saade Rodrigues ${ }^{1 *}$, Bruna Cristina Silva Martins ${ }^{1}$, Daniel Caires Campos ${ }^{2}$, Helena Maia Ferreira de Paula Medeiros ${ }^{3}$, João Marcelo Feitoza de Andrade ${ }^{4}$, Lorenna Lemos de Aquino ${ }^{5}$, Maria Alice Batista Rocha ${ }^{6}$, Maria Eduarda Venturim Almeida Vieira ${ }^{7}$, Maria Gabriela Prandini Nunes Cota ${ }^{1}$, Gabriela Gonçalves Campos ${ }^{8}$.

\section{RESUMO}

Objetivo: Elaborar uma revisão narrativa da literatura com a finalidade de relatar as principais manifestações clínicas e os desfechos gestacionais até o momento em gestantes com COVID-19 (infecção respiratória aguda causada pelo coronavírus SARS-CoV-2). Revisão bibliográfica: A gravidez acarreta várias mudanças fisiológicas e imunológicas, a qual o corpo fica mais suscetível a infecções por patógenos respiratórios e desenvolvimento de pneumonias graves, que é considerada a terceira causa indireta mais frequente de morte materna. O quadro clínico em gestantes com COVID-19 é capaz de se manifestar assintomática, bem como de forma sintomática grave, necessitando de tratamento em unidade de terapia intensiva. Partos prematuros, pneumonia neonatal e transmissão vertical foram associados a bebês de mães infectadas pelo COVID-19. Considerações finais: Isto posto, torna-se necessário um cuidado especial por parte da equipe com a gestante. Entretanto, o isolamento social é a melhor forma de combater o vírus e evitar suas complicações, uma vez que a imprevisibilidade dessa doença pode ocasionar um desfecho agressivo para o feto, além de complicações severas para a mãe.

Palavras-chave: Complicações na gravidez, COVID-19, Saúde da mulher.

\begin{abstract}
Objective: To prepare a narrative review of the literature in order to report the main clinical manifestations and pregnancy outcomes to date in pregnant women with COVID-19 (acute respiratory infection caused by the SARS-CoV-2) coronavirus. Bibliographic review: Pregnancy causes several physiological and immunological changes, which the body is more susceptible to infections by respiratory pathogens and development of severe pneumonia, which is considered the third most frequent indirect cause of maternal death. The clinical picture in pregnant women with COVID-19 is capable of manifesting itself asymptomatic, as well as severely symptomatically, requiring treatment in an intensive care unit. Premature births, neonatal pneumonia and vertical transmission have been associated with babies of mothers infected with COVID-19. Final considerations: That said, it is necessary a special care by the team with the pregnant woman. However, social isolation is the best way to fight the virus and avoid its complications, since the unpredictability of this disease can lead to an aggressive outcome for the fetus, in addition to severe complications for the mother.
\end{abstract}

Keywords: Pregnancy complications, COVID-19, Women's health.

\footnotetext{
${ }^{1}$ Pontifícia Universidade Católica de Minas Gerais (PUC Minas), Betim - MG.

*E-mail: gabrieltorga1@gmail.com

${ }^{2}$ Centro Universitário de Brasília (CEUB), Brasília - DF.

${ }^{3}$ Centro Universitário de Volta Redonda (UniFOA), Volta Redonda - RJ.

${ }^{4}$ Faculdade de Ciências Médicas da Paraíba (FCMPB), João Pessoa - PB.

${ }^{5}$ Universidade de Rio Verde (UniRV), Goianésia - GO.

${ }^{6}$ Centro Universitário Facisa (Unifacisa), Campina Grande - PB.

${ }^{7}$ Centro Universitário UNIFAMINAS, Muriaé - MG.

${ }^{8}$ Centro Universitário de Caratinga, Caratinga - MG.
} 


\section{RESUMEN}

Objetivo: Elaborar una revisión narrativa de la literatura con el fin de reportar las principales manifestaciones clínicas y resultados del embarazo hasta la fecha en mujeres embarazadas con coronavirus COVID-19 (infección respiratoria aguda causada por el SARS-CoV-2). Revisión bibliográfica: El embarazo provoca diversos cambios fisiológicos e inmunológicos, por lo que el organismo es más susceptible a infecciones por patógenos respiratorios y al desarrollo de neumonía grave, que se considera la tercera causa indirecta más frecuente de muerte materna. El cuadro clínico en mujeres embarazadas con COVID-19 es capaz de manifestarse asintomático, así como severamente sintomático, requiriendo tratamiento en una unidad de cuidados intensivos. Los nacimientos prematuros, la neumonía neonatal y la transmisión vertical se han asociado con bebés de madres infectadas con COVID-19. Consideraciones finales: Dicho esto, es necesario un cuidado especial por parte del equipo con la embarazada. Sin embargo, el aislamiento social es la mejor forma de combatir el virus y evitar sus complicaciones, ya que la imprevisibilidad de esta enfermedad puede conllevar un desenlace agresivo para el feto, además de graves complicaciones para la madre.

Palabras clave: Complicaciones del embarazo, COVID-19, Salud de la mujer.

\section{INTRODUÇÃO}

Em dezembro de 2019, a Organização Mundial de Saúde (OMS) identificou relatos sobre casos atípicos de pneumonia na cidade de Wuhan, China. No decorrer de janeiro de 2020, foi confirmado pelas autoridades chinesas, que os casos foram causados pela mutação do vírus da família coronavírus. Com o aumento do número de casos relatados ao redor do mundo, em março do ano de 2020, a OMS caracteriza como pandemia o vírus SARS-Cov-2, também conhecido como COVID-19 (ORGANIZAÇÃO MUNDIAL DA SAÚDE, 2020). Ao decorrer do estudo do novo vírus, observou-se que pessoas previamente acometidas com doenças crônicas apresentavam risco aumentado para o desenvolvimento grave da doença e as gestantes foram posteriormente adicionadas neste grupo de risco (AVILA WS e CARVALHO RC, 2020).

Por se tratar de uma mutação recente do vírus, há grande escassez de estudos com informações conclusivas de como a nova alteração do vírus está afetando a saúde de gestantes. A revisão sistemática feita por Trad ATA, et al. (2020) apontou que 98,6\% das contaminações ocorreram no terceiro trimestre, sendo que $87,7 \%$ das pacientes do estudo possuíam uma gravidez de baixo risco. Ao analisar o estudo de Castro $\mathrm{P}$, et al. (2020) observa-se que se comparado com outros vírus da família coronavírus, o COVID-19 apresenta melhor desfecho final da doença. A taxa de mortalidade materna reportada para SARS-CoV e MERS-CoV foram de, respectivamente, $28,6 \%$ e $25,8 \%$, para COVID-19 e não foram reportadas, no estudo, mortes maternas.

Considerando que gestantes apresentam propensão aumentada para complicações de infecções devido a alterações fisiológicas durante a gravidez, foi recomendado a essa população maior cuidado durante a pandemia. Durante a gravidez, as alterações fisiológicas como: alterações dos volumes pulmonares, elevação do diafragma, alterações na imunidade mediada por células, entre outras, podem colocar essa população em condição de risco para infecções respiratórias. Também foi relatado que as alterações anatômicas podem reduzir a tolerância à hipóxia (MINISTÉRIO DA SAÚDE, 2020b).

As manifestações clínicas das gestantes com COVID-19 diversificam-se em sintomas graves a pacientes assintomáticas, similar ao que ocorre na população em geral. Os principais sintomas em gestantes são febre, tosse e fadiga. Além desses, também estão presentes sintomas como dispneia, dor de garganta, mialgia e diarreia, entretanto em menor proporção. Um pequeno número de casos evoluiu para pneumonia grave necessitando de tratamento em unidade de tratamento intensivo (YAN J, et al., 2020).

Referente às gestantes infectadas com COVID-19, evidenciaram que as informações sobre o desfecho gestacional ainda são limitadas, entretanto, sugerem risco aumentado de parto prematuro e baixo peso ao nascer dos recém-nascidos. Além disso, alguns estudos apontam que a transmissão vertical de COVID-19 para recém-nascidos é possível, mas os indícios ainda são inconclusivos (MELO GC e ARAÚJO KCGM, 2020).

A presente revisão teve por objetivo a elaboração de uma revisão narrativa da literatura com a finalidade de contextualizar a gestação no atual cenário de pandemia e de relatar as principais manifestações clínicas, 
as alterações fisiológicas maternas, o manejo das gestantes e o desfecho gestacional até o momento, ocasionados pela infecção por COVID-19, a fim de aprimorar a assistência à saúde da gestante nesse cenário de pandemia do novo coronavírus.

\section{REVISÃO BIBLIOGRÁFICA}

\section{Gestantes como grupo de risco}

No contexto da pandemia do COVID-19, a perspectiva inicial era de que a infecção pelo SARS-CoV-2 não possuía relação com complicações gestacionais ou durante o parto. Todavia, recentes mudanças nos dados maternos, correspondentes com o acometimento pelo novo coronavírus, mudou completamente a condução desses quadros (FAIRLIE L, et al., 2021). Após aumento da mortalidade materna no Brasil, as gestantes passaram a ser classificadas como grupo de risco sendo, inclusive, realocadas como população prioritária para o recebimento das doses de vacina já disponíveis contra o vírus (MINISTÉRIO DA SAÚDE, 2021).

A gravidez ocasiona alterações fisiológicas e imunológicas que expõem as gestantes a maiores riscos de infecções por patógenos respiratórios e desenvolvimento pneumonias graves. Fatores como expansão do útero, elevação diafragmática, aumento do consumo de oxigênio e edema da mucosa do trato respiratório contribuem para aumento da percepção subjetiva de falta de ar e se relacionam com a intolerância à hipóxia presente nas grávidas. Além disso, a modulação sofrida pelo sistema imune o para evitar a rejeição do feto coloca a mãe em uma situação de imunossupressão relativa, tornando estas mulheres ainda mais vulneráveis a infecções em geral (CASTRO P, et al., 2020; BOUSHRA MN, et al, 2021).

Relatos de partos prematuros, pneumonia neonatal e transmissão vertical e pós-parto infecções foram associados a bebês de mães infectadas pelo COVID-19. Além disso, a associação entre mudanças hemodinâmicas no pós-parto e o caráter trombogênico tanto da gravidez quanto da doença, pode ter sido o principal fator de algumas mortes maternas. Segundo Castro P, et al. (2020), as pneumonias são a terceira causa indireta mais frequente de morte materna e exigem suporte ventilatório em pelo menos $25 \%$ dos casos. Por fim, ruptura prematura de membranas, natimorto, restrição de crescimento intrauterino e prematuridade são complicações comuns de acometimento pulmonares (FAIRLIE L, et al., 2021; CASTRO P, et al., 2020)

\section{Manifestações clínicas}

As gestantes não apresentam predileção a serem infectadas comparadas a população geral. A maioria das gestantes infectadas com SARS-CoV-2 apresentam quadro assintomático. Quando sintomáticas, há predominância a sintomas leves ou moderados característicos de resfriado/ gripe, sendo que os sintomas mais recorrentes são: tosse $(64 \%)$ e febre $(68 \%)$. Outros sintomas menos frequentes também foram relatados, como mal-estar (13\%), dispnéia (12\%), mialgia (19\%), disgeusia (14\%) e diarreia (6\%) (CASTRO P, et al., 2020).

De acordo com um estudo comparativo entre gestantes sem COVID-19 e com COVID- 19 sintomáticas que requerem hospitalização realizado por Cardoso PC, et al. (2021), as grávidas com quadro agudo apresentam piora do quadro geral, incluindo risco de morte aumentado. A frequência de evolução em gestantes diagnosticadas com COVID-19 varia de infecção assintomática a doença leve, com ausência de hipóxia ou pneumonia, doença moderada quando apresenta quadro de pneumonia viral e doença grave, necessitando de tratamento em unidade de terapia intensiva (YAN J, et al., 2020). O quadro geral ressalta acometimento por grave pneumonia, sendo frequente $\mathrm{SpO} 2$ menor que 90\% em ar ambiente e presença de manifestações críticas, comumente Síndrome de Angústia Respiratória Crítica (SDRA), sepse, choque séptico ou complicações envolvendo embolia pulmonar ou síndrome coronariana aguda (BOUSHRA MN, et al., 2021).

\section{Manejo das gestantes}

A padronização do manejo das gestantes, na situação da pandemia, gera inúmeras controvérsias devido a inexistência de um tratamento eficaz para a covid-19 e a falta de estudos da segurança da vacina em grávidas. Porém, há um consenso de que o manejo deve priorizar a redução da transmissão viral, a adequação da oferta de atendimentos e procedimentos, além do tratamento sintomático das manifestações. (GOYAL M, 2020; ORTIZ EI, 2020). 
A medida básica de redução da transmissão viral é o isolamento, no qual os atendimentos são realizados por telessaúde, por consulta nas unidades básicas de saúde e por visitas domiciliares, sendo necessária a internação para as gestantes de alto risco obstétrico-neonatal com a doença em caso leve e para as demais gestantes com a doença moderada ou grave. Os hospitais e as maternidades adotaram ações para vigilância em saúde, como determinação precoce do fluxo de atendimento, isolamento das grávidas internadas e decisões em conjunto pela equipe multidisciplinar (FERNÁNDEZ ABS, 2020; MINISTÉRIO DA SAÚDE, 2020b; MINISTÉRIO DA SAÚDE, 2020c).

Ressalta-se que as consultas devem buscar a história pregressa da paciente, atentando-se ao contato com pessoas infectadas e ao histórico da vacinação contra influenza, as manifestações clínicas mais comuns na infecção por coronavírus e aos tratamentos realizados, para a tomada da conduta mais adequada, a ser julgada pelo médico (CAMUS FA, 2020). O manejo das gestantes é individualizado, a depender da sintomatologia e da gravidade da doença. A falta de consenso sobre as medicações a serem utilizadas é desafiadora, por isso visa-se o tratamento da sintomatologia. Há divergências sobre o uso de antibióticos e outros medicamentos profiláticos para pneumonia, durante o tratamento do paciente com COVID-19. Nos casos de internação, os cuidados gestacionais usuais devem ter a vigilância aumentada, com o devido controle da monitoração da contração uterina, da oxigenação materna e dos sinais vitais (WU B, et al, 2020; FURLAN MCR, et al., 2020; MINISTÉRIO DA SAÚDE, 2020c).

Os atendimentos também devem priorizar as orientações dadas às gestantes, enfatizando que qualquer agravamento do quadro deve ser comunicado, para a avaliação, por um profissional da saúde, e possível encaminhamento (MINISTÉRIO DA SAÚDE, 2020b). Cardoso PC, et al. (2021), explica que o esclarecimento das condutas ajuda no manejo da saúde mental das gestantes, sendo recomendado o apoio psicológico para a paciente e o seu acompanhante.

\section{Direito a acompanhante, cuidados da equipe, parto, amamentação e pós-parto}

Toda gestante e seu acompanhante devem ser triados para COVID-19 antes da admissão no serviço obstétrico. Será considerado suspeito ou confirmado aquele que esteve em contato com caso suspeito ou confirmado, apresentar febre ou sintoma respiratório e também aquele que apresentar resultado de exame positivo para SARS-CoV-2 nos últimos 14 dias. Gestantes suspeitas ou confirmadas têm o direito a acompanhante, desde que assintomático, fora dos grupos de risco para COVID-19 e que seja de convívio diário da paciente. Não é permitido revezamento e os acompanhantes deverão ficar restritos ao local de assistência. Além disso, deverão permanecer com máscara cirúrgica e manter distanciamento de um metro da paciente (MINISTÉRIO DA SAÚDE, 2020a).

As unidades obstétricas que receberem casos suspeitos ou positivos devem ser informadas previamente a chegada de tais pacientes para fim de preparação e implementação de medidas de biossegurança. O parto deverá ocorrer, preferencialmente, em salas reservadas, com medidas rigorosas de higiene e equipamentos de proteção individual. A equipe de parto deve incluir um número mínimo de profissionais e evitar a circulação em outros ambientes. Todos profissionais envolvidos na assistência a paciente, devem seguir rígido protocolo de proteção pessoal, usando toucas, capotes, máscaras N95/PFF2, óculos de proteção ou protetores faciais e luvas, além de realizar higiene das mãos antes e depois de sair da sala (MASCARENHAS VHA, et al., 2020).

Até o momento, não há contraindicação para partos vaginais se as avaliações clínica e obstétrica forem favoráveis. Sugere-se escolha com base em indicações obstétricas e preferências da mulher, sendo a realização da cesariana idealmente indicada quando houver justificativa, dentre elas a instabilidade clínica ou obstétrica, além de alterações na vitalidade fetal. O parto na água é contraindicado pela possibilidade de contaminação da água por fezes, sangue e urina da mãe e, consequentemente, elevado risco de transmissão para o RN (CARDOSO PC, et al., 2021; MASCARENHAS VHA, et al., 2020).

A analgesia axial deve ser oferecida quando apropriado e de acordo com o desejo da paciente. Não há evidências de aumento da transmissão do vírus sob punção raquidiana e/ou peridural. Porém, a anestesia geral deverá ser evitada, pois o procedimento da intubação orotraqueal gera aerossóis que aumentam o risco 
de contaminação do ambiente. Métodos não farmacológicos de alívio da dor podem e devem ser oferecidos, pois favorecem a evolução fisiológica do parto. Em pacientes confirmadas assintomáticos ou com doença leve, foi possível realizar a indução do parto de acordo com os protocolos padrão, com taxas de sucesso semelhantes aos sem a doença (JÚNIOR AT, et al., 2020; BOCIC NC, et al., 2020).

Ainda há discrepância entre as orientações para o clampeamento tardio do cordão umbilical, em função de falta de evidências. O Colégio Real de Obstetras e Ginecologistas recomenda que o clampeamento retardado do cordão seja praticado normalmente sob justificativa de que o parto vaginal causa exposição a secreções maternas e sangue, logo, 1 minuto de perfusão adicional provavelmente não alterará o risco de transmissão vertical. A Sociedade Brasileira de pediatria também recomenda que o clampeamento oportuno deve ser mantido (MULLINS E, et al., 2020).

Até o momento, não há constatação científica que estabeleça nexo causal entre a transmissão do SARSCoV-2 e a amamentação, sendo recomendada para pacientes positivas, desde que se encontre em condição clínica satisfatória e assim o deseje. A maior preocupação reside na possibilidade de contaminação do RN pelas gotículas respiratórias. Para diminuir esse risco, recomenda-se a troca de máscara, touca, camisola e lençóis, mas que idealmente ocorra dentro da $1^{\underline{a}}$ hora de nascimento ou tão precocemente possível. Durante cada mamada, a mãe deverá fazer uso de máscaras cirúrgicas e higienizar adequadamente as mãos. Se a paciente optar por não amamentar, os profissionais de saúde devem incentivá-las a bombear o leite materno para ser ofertado ao bebê por cuidador saudável, seguindo os cuidados higiênicos preconizados (PAZ MMS, et al., 2021; SOCIEDADE BRASILEIRA DE PEDIATRIA, 2020).

Caso a mãe esteja clinicamente estável e RN assintomático, o alojamento conjunto poderá ser mantido, desde que respeite a distância de dois metros entre o leito da mãe e o berço do $\mathrm{RN}$, faça uso de máscara cirúrgica e lave as mãos antes de tocar o RN. A alta hospitalar deve ser realizada o mais precocemente possível, respeitando as condições clínicas de mãe e RN, em 24 e 48 horas após o parto normal e cesárea, respectivamente (JUNIOR AT, et al., 2020).

\section{Prognóstico}

Primeiramente, é necessário salientar que gestantes em qualquer idade gestacional e puérperas até 2 semanas após a gravidez (incluindo casos de aborto ou perda fetal), são consideradas grupos de risco quando tratamos da infecção pelo coronavírus. No entanto, também está claro que os sintomas em mulheres grávidas sem comorbidades se assemelham muito aos sintomas em mulheres não grávidas, sendo febre e tosse os principais sintomas, e o prognóstico desses pacientes é considerado bom. É importante salientar que se trata de uma doença bastante imprevisível, e que uma possível infecção possa ocasionar desfechos obstétricos extremamente desfavoráveis como sofrimento fetal, aborto espontâneo, dificuldade respiratória, além do aumento da necessidade de parto cirúrgico (CARDOSO PC, et al., 2021).

Existem variações nas manifestações clínicas registradas nas pacientes grávidas e que contraíram o COVID-19, alternando desde casos assintomáticos, até casos mais graves, com necessidade de ventilação mecânica. Uma das formas de exame que houve um maior número de respostas precisas sobre a infecção, foi através da tomografia computadorizada de tórax (TCT), que permitiu a visualização de opacidade e consolidação parcial na região pulmonar, ajudando a diagnosticar clinicamente tais pacientes, visto que existem casos assintomáticos e esses casos precisam sim de acompanhamento a fim de evitar desfechos obstétricos desfavoráveis (FURLAN MCR, et al., 2020).

Quando pensamos em gestantes cardiopatas, há um maior risco de complicações cardíacas graves, sendo extremamente necessário um acompanhamento de perto por parte da equipe. A COVID-19 é marcado pela presença de uma infecção sistêmica grave e falência múltipla de órgãos, sendo assim o sistema cardiovascular também é afetado, diante disso pacientes que já são cardiopatas possuem um risco ainda mais aumentado, podendo causar lesões miocardicas, infarto aguda do miocárdio, insuficiência cardíaca, arritmias e eventos tromboembólicos. É importante salientar, portanto, que medidas protetivas que busquem tratamento individualizado de acordo com a estratificação de risco para cardiopatias é de extrema importância para um bom prognóstico tanto para mãe quanto para o feto (SANTOS CL, et al., 2020). 


\section{CONSIDERAÇÕES FINAIS}

A partir da análise do presente artigo, verificou-se que a Covid-19 em mulheres grávidas ainda é campo de incertezas. Há uma certa limitação sobre o assunto, devido à escassez de pesquisas robustas e evidências sólidas. Dessa forma, demonstra-se a necessidade de estudos mais efetivos, que consigam apurar com clareza quais os efeitos da COVID 19 na gestação. No presente momento, foram documentados diversos desfechos gestacionais, com uma diversidade de sinais e sintomas associados, e por isso é necessário que o manejo das gestantes com coronavírus seja individualizado. Além disso, foi concluído que grávidas são grupo de risco na doença, portanto, isolamento social, uso de máscara e higienização das mãos com álcool em gel se fazem mister na prevenção do COVID, principalmente para o grupo supracitado.

\section{REFERÊNCIAS}

1. AVILA WS, CARVALHO RC. COVID-19: Um Novo Desafio para a Cardiopatia na Gravidez. Arquivos Brasileiros de Cardiologia, 2020; 115(1): 1-4.

2. BOUSHRA MN, et al. COVID-19 in pregnancy and the puerperium: A review for emergency physicians. The American Journal of Emergency Medicine, USA, 2021; 40: 193-198.

3. BOCIC NC, et al. Inducción de trabajo de parto en pacientes COVID-19: experiencia en el Hospital San Juan de Dios. Revista Chilena de Obstetrícia e Ginecologia, 2020; 85(1): 28-34.

4. CAMUS FA. COVID-19 y Embarazo. Revista Chilena de Obstetricia y Ginecología. 2020; 85(2): 110-114.

5. CARDOSO PC, et al. A saúde materno-infantil no contexto da pandemia de COVID-19: evidências, recomendações e desafios. Revista Brasileira de Saúde Materno Infantil, 2021; 21 (Supl.1): S221-S228.

6. CASTRO P, et al. Covid-19 and Pregnancy: An Overview. Revista Brasileira de Ginecologia e Obstetrícia, 2020; $42(7)$ : 420426

7. FAIRLIE L, et al. COVID-19 in pregnancy in South Africa: tracking the epidemic and defining the natural history. S AfrMed J. Johannesburg, 2020; 110 (8): 729 - 731.

8. FERNÁNDEZ ABS. Clinical characterization of obstetric patients with suspected COVID-19. Revista Cubana de Medicina Militar, 2020; 49(3): e0200796.

9. FURLAN MCR, et al. Gravidez e infecção por Coronavírus: desfechos maternos, fetais e neonatais. Revista Cuidarte, 2020; 11(2): e1211.

10. GOYAL M, et al. Review of care and management of pregnant women during COVID-19 pandemic. Taiwanese Journal of Obstetrics \& Gynecology. 2020, 59: 791-794.

11. JÚNIOR AT, et al. Childbirth, Puerperium and Abortion Care Protocol during the COVID-19 Pandemic. Revista Brasileira de Ginecologia e Obstetrícia, 2020; 42(6): 349-355.

12. LISTINGS OF WHO'S RESPONSE TO COVID-19. 2020. In: World Health Organization. Disponível em: https://www.who.int/news/item/29-06-2020-covidtimelineAcessoem: 26 abr. 2021.

13. MASCARENHAS VHA, et al. Care recommendations for parturient and postpartum women and newborns during the COVID19 pandemic: a scoping review. Revista Latino Americana de Enfermagem. 2020; 28: e3359.

14. MELO GC, ARAÚJO KCGM. COVID-19 infection in pregnant women, preterm delivery, birth weight, and vertical transmission: a systematic review and meta-analysis. Cad. Saúde Pública, 2020; 36(7): e00087320.

15. MINISTÉRIO DA SAÚDE. Manual de Recomendações para a Assistência À Gestante e Puérpera frente à Pandemia de Covid-19. 2020c. Disponível em: http://antigo.saude.gov.br/images/pdf/2020/September/02/Manual-de-Recomenda----espara-Gestante.pdf. Acesso em: 01 de maio de 2021.

16. MINISTÉRIO DA SAÚDE. Nota Técnica № 1/2021-DAPES/SAPS/MS. Tratam-se das recomendações referentes a administração de vacinas covid-19 em gestantes, puérperas e lactantes, incluindo os esclarecimentos que devem ser fornecidos para tomada de decisão, 2021. Disponível em: https://portaldeboaspraticas.iff.fiocruz.br/wpcontent/uploads/2021/03/NT-vacinacao-gestantes-peurperas-e-lactantes.pdf. Acesso em: 31 de maio de 2021.

17. MINISTÉRIO DA SAÚDE. Nota Técnica № 9/2020-COSMU/CGCIVI/DAPES/SAPS/MS. Recomendações para o trabalho de parto, parto e puerpério durante a Pandemia da Covid-19. Brasil, 2020a. Disponível em: https://portaldeboaspraticas.iff.fiocruz.br/wp-content/uploads/2020/04/SEI_MS-0014382931-NotaTecnica 9.4.2020 parto.pdf. Acessado em: 31 de maio de 2021.

18. MINISTEERIO DA SAÚDE. Secretaria de Atenção Primária à Saúde. Nota Técnica № 12/2020COSMU/CGCIVI/DAPES/SAPS/M. Infecção COVID-19 e os riscos às mulheres no ciclo gravídico-puerperal. Brasil, $2020 \mathrm{~b}$. Disponível em: https://portaldeboaspraticas.iff.fiocruz.br/biblioteca/nota-tecnica-no-12-2020-cosmu-cgcivi-dapes-saps-ms/. Acesso em: 26 abr. 2021.

19. MULLINS E, et al. Coronavirus in pregnancy and delivery: rapid review. Ultra sound in Obstetrics \& Gynecology, 2020; 55(5): 586-592.

20. ORTIZ El, et al. Coronavirus (COVID-19) Infection in Pregnancy. Colombia Médica. 2020; 51(2): e-4271.

21. PAZ MMS, et al. Barreiras impostas na relação entre puérperas e recém-nascidos no cenário da pandemia do COVID-19. Revista Brasileira de Saúde Materno Infantil, 2021; Recife, 21(Supl. 1): S233-S236.

22. SOCIEDADE BRASILEIRA DE PEDIATRIA (SBP). Departamento Científico de Aleitamento Materno. Nota de Alerta, Aleitamento Materno em tempos de COVID-19 - recomendações na maternidade e após a alta. Brasil, 2020. Disponível em: https://www.sbp.com.br/fileadmin/user_upload/22467f-NA_-_AleitMat_tempos_COVID-19-_na_matern_e_apos_alta.pdf. Acessado em: 31 de maio de 2021.

23. TRAD ATA, et al. Complications and outcomes of SARS-CoV-2 in pregnancy: where and what is the evidence?. Hypertension in pregnancy,2020; 39(3): 361-369.

24. WU B, et al. Strategic plan for the management of COVID-19 in an obstetrics department. Revista da Associação Médica Brasileira. 2020; 66(7):890-893.

25. YAN J, et al. Coronavirus disease 2019 in pregnant women: a report based on 116 cases. Am J Obstet Gynecol, 2020; 23: 111.e1-14. 